\title{
Política tributaria y fiscal del Ecuador
}

\author{
Tax and fiscal policy of Ecuador
}

Lenin Agustín Chamba Bastidas. ${ }^{1}$, Raquel Virginia Colcha Ortiz. ${ }^{2}$, María del Carmen Moreno Albuja. ${ }^{3} \&$ Marco Antonio Gavilanes Sagñay. ${ }^{4}$

\section{DOI: https://doi.org/10.33262/visionariodigital.v3i3.886}

\begin{abstract}
Fiscal policy is a fundamental tool in public management and economic science of Ecuador, the economic stability of the country depends largely on this policy, an important reason why it is necessary to carry out analyzes that allow contextualizing its planning, execution and results.

In this paper we want to analyze the relationship between inflation and employment, according to Phillips, the relationship is inversely proportional, according to the global economy, the relationship is not evidenced that meets that characteristic, on the contrary, a relationship with trends is observed direct.

Likewise, the relationship between public spending and fiscal pressure, its analysis is also necessary, which mainly shows that from 1993 to 1999, public spending is higher and as of 2000, the fiscal pressure is the highest.
\end{abstract}

Keywords: Fiscal policy, Ecuador, Economy.

\section{Resumen}

La política fiscal es una herramienta fundamental en la gestión pública y la ciencia económica del Ecuador, la estabilidad económica del país depende en gran medida de esta política, razón importante por la cual es necesario realizar análisis que permitan contextualizar su planificación, ejecución y resultados.

En el presente trabajo queremos analizar la relación que se presenta entre la inflación y el empleo, según Phillips la relación es inversamente proporcional, según The global economy la relación no se evidencia que cumpla con aquella característica, al contrario, gráficamente se observa una relación con tendencia directa.

Así mismo la relación entre el gasto público y la presión fiscal, también es necesario su análisis, del cual se desprende principalmente que a partir de 1993 hasta 1999, el gasto público es mayor y a partir del 2000, es la presión fiscal la mayor.

\footnotetext{
${ }^{1}$ Escuela Superior Politécnica de Chimborazo, Facultad de Administración de Empresas, Riobamba, Ecuador, lenin.chamba@espoch.edu.ec

${ }^{2}$ Escuela Superior Politécnica de Chimborazo, Facultad de Administración de Empresas, Riobamba, Ecuador, raquel.colcha@espoch.edu.ec

${ }^{3}$ Escuela Superior Politécnica de Chimborazo, Facultad de Administración de Empresas, Riobamba, Ecuador, Carmen.moreno@espoch.edu.ec

${ }^{4}$ Escuela Superior Politécnica de Chimborazo, Facultad de Administración de Empresas, Riobamba, Ecuador, marco.gavilanes@espoch.edu.ec
} 
Palabras Claves: Política fiscal, Ecuador, Economía.

\section{Introducción}

La política fiscal es una rama de la política económica y se la utiliza como herramienta para influir de manera positiva en la economía de un determinado país mediante la carga impositiva o los impuestos, busca también mejorar la redistribución de la riqueza, disminuir la pobreza y en sí, mejorar el nivel de vida de sus habitantes sin que la producción sufra un efecto negativo, caso contrario sería una medida contraproducente ya que no cumpliría con el fin económico propuesto.

El Ecuador, es un país que desde el punto de vista económico y en gran medida, depende de productos renovables y no renovables que nos ofrece la naturaleza; la industria y los servicios tienen un aporte relativamente mínimo a comparación de otros países principalmente del norte de América y de Europa. Los auges de productos naturales que a lo largo de la historia hemos tenido, corroboran que lamentablemente no hemos desarrollado ventajas absolutas, ni siquiera en los productos naturales, lo cual implica estar en un estado de confort para quienes gobiernan el país y un estado de pobreza relativa a la gente que vive en el Ecuador, si bien el coeficiente de la brecha de pobreza (Banco Mundial) es menor a 2 para el año 2014, no es admisible que cierto grupo de individuos sigan viviendo con 2 dólares por día.

Otra característica importante a resaltar, es la deuda pública ${ }^{5}$, misma que fue muy representativa entre 1999 y 2000; luego de dicho periodo, la tendencia es a disminuir hasta el año 2012, posterior a ello, la tendencia es a crecer. La Constitución del Ecuador del año 2008 y demás normativa, regulan el endeudamiento, principalmente en el artículo 290 de la Constitución, por lo que se esperaría que el endeudamiento no llegue a niveles de décadas pasadas.

Si bien, en la actualidad podemos observar y evidenciar que, los tributos, y de manera especial, los impuestos han sufrido una mejor y efectiva recaudación en comparación con años anteriores a la existencia de la actual administración central del Servicio de Rentas

\footnotetext{
${ }^{5}$ Conjunto de títulos valores emitidos por el Estado, otros poderes públicos o sus organismos autónomos, como forma de captar recursos financieros. El interés que debe pagar a los poseedores de dichos títulos (bonos, pagarés, letras, etc.) supone uno de sus mayores gastos anuales (Arthur Andersen , 1999)
} 
Internas; se corrobora lo descrito en párrafos anteriores, el nivel de vida de los individuos que viven en el Ecuador, no es necesariamente el adecuado ni el mejor comparado con los que están por encima de la media en la región. La distancia se hace más grande, cuando comparamos con otros países que tienen mejor calidad de vida como los indicados la ONG Social Progress Imperative.

Los recursos que obtiene el Estado, deben servir para mitigar la pobreza y con mayor énfasis en la pobreza extrema que no está eliminada por completo. El sistema económico que se maneja a partir del 2008 con los cambios que se dieron en la Constitución y del cual resalto la parte del artículo 275, en el que se indica: El Estado planificará el desarrollo del país (Constitución de la República de Ecuador, 2008) “ el Ecuador tiene mejoría en ciertos indicadores económicos, los cuales no son suficiente, en principio es necesario seguir la misma línea de planificación con miras a tener resultados sostenidos y sostenibles. Los buenos resultados no pueden ser temporales, por ese motivo hay que pensar en el futuro para la obtención de recursos que permitirán satisfacer las necesidades colectivas.

\section{Desarrollo}

Es necesario que iniciemos definiendo la política fiscal e ir introduciéndonos también a la teoría, para comparar las variables macroeconómicas, emparejar y comparar los resultados obtenidos en el Ecuador y concluir con un análisis económico.

La política fiscal es una rama de la política económica que configura el presupuesto del Estado, y sus componentes, el gasto público y los impuestos, como variables de control para asegurar y mantener la estabilidad económica, amortiguando las variaciones de los ciclos económicos, y contribuyendo a mantener una economía creciente, de pleno empleo y sin inflación alta. El nacimiento de la teoría macroeconómica keynesiana puso de manifiesto que las medidas de la política fiscal influyen en gran medida en las variaciones a corto plazo de la producción, el empleo y los precios (Wikipedia, 2008).

Conjunto de medidas y/o actuaciones tomadas por el Gobierno o entidades con capacidad regulatoria en la materia (como las Comunidades Autónomas) con la finalidad de influir en el nivel de precios, en la producción, en la inversión y en el empleo. Si lo que se persigue es salir de una situación de depresión con altas tasas de paro, sería necesaria la aplicación de una política fiscal expansiva mediante un 
incremento del gasto público o de una reducción de los impuestos principalmente, pero si lo que se quiere es combatir una situación inflacionista, habría que aplicar una política fiscal restrictiva (Andersen, A., 1999).

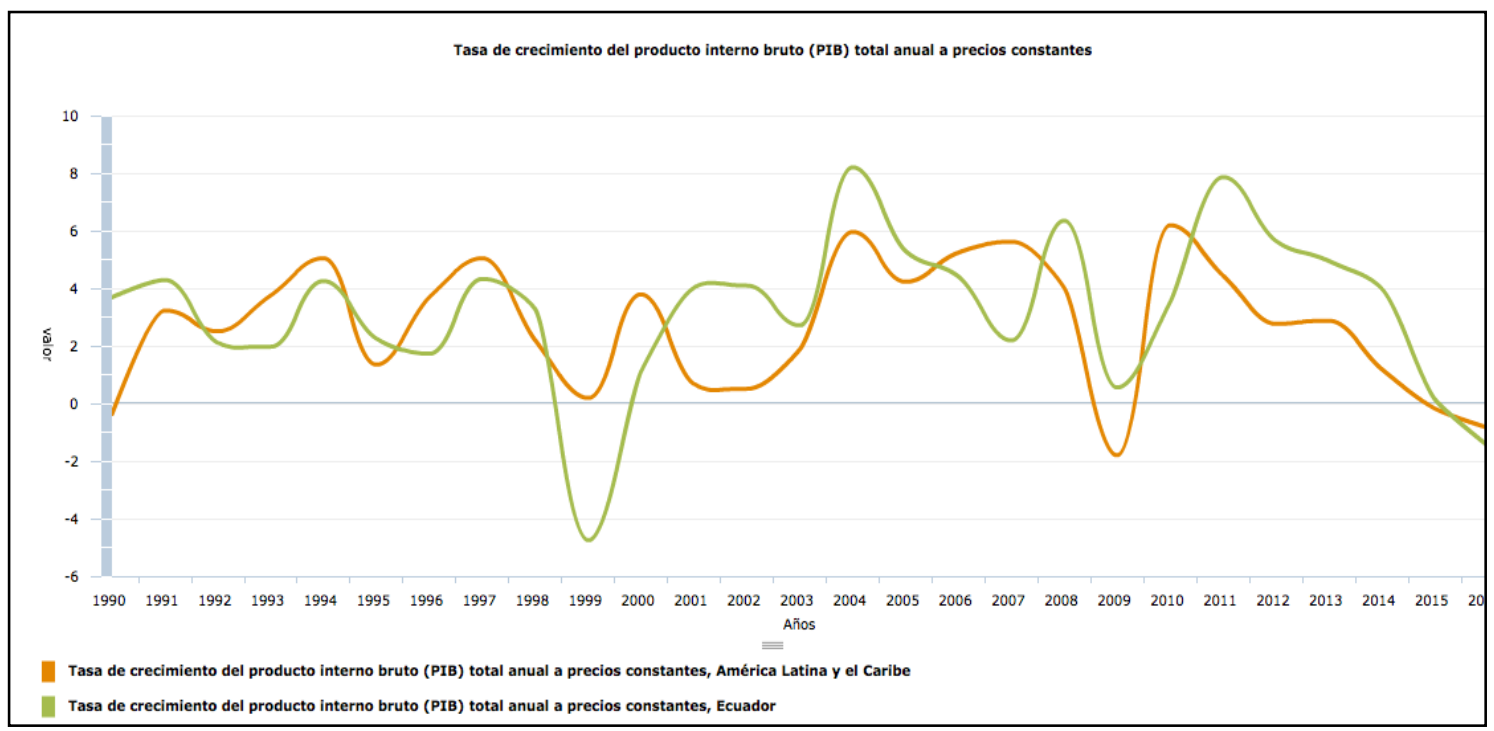

Figura 1. Variación del PIB a precios constantes $(1990$ - 2016)

Fuente: CEPAL (https://www.cepal.org/es)

Considerando la primera definición de política fiscal y el gráfico uno, podemos evidenciar que los ciclos económicos son representativos, tanto en crecimiento cuando en el decrecimiento, están bastante relacionados con el comportamiento que se presente a nivel de América Latina y el Caribe, salvo la gran diferencia que se observa en el año 1999, año en el cual se produjo la mayor crisis del Ecuador en el período objeto de análisis.

Entre 1990 y 1997, las variaciones son pequeñas y de alguna manera están en el cuadrante positivo, lo que significa que la afectación cuando la pendiente es negativa, no afecta como en el siguiente ciclo que inicia en 1998 y 1999; período extremadamente negativo para la economía del Ecuador, su decrecimiento es el más grande al menos en el período del presente análisis, situación que a priori, podemos decir obedece principalmente a los cambios en el manejo económico - financiero, la crisis financiera internacional, conflicto bélico con el Perú, el fenómeno del niño y los intereses particulares de los gobernantes de turno.

Se observa una leve similitud de comportamiento de la curva entre América Latina y el Caribe con relación a Ecuador de manera general, como también una clara diferencia en el 
año 98 - 99, la curva de A.L. y el Caribe no baja al cuadrante negativo como si lo hace el Ecuador por las causas descritas en líneas generales.

El siguiente ciclo es creciente, el más alto para Ecuador en el período, incluso más alto que el de A.L y el Caribe, si bien la dolarización que para el año 2004 ya estaba en completa vigencia, no fue la única responsable de dicho resultado positivo.

Para el 2009, cambia la tendencia de positiva a negativa, acercándonos al cuadrante negativo, sin embargo A.L. y el Caribe, pasan al cuadrante negativo, siendo esta la mayor crisis para dicho periodo, misma que obedece a la caída de las exportaciones tanto en precio como en volumen. Sin duda, una crisis internacional afecta significativamente la economía de nuestra región.

El Ecuador toma un repunte entre al año 2011 y 2012, faltando menos de un punto para igualar al ciclo positivo del 2004, el segundo más grande en el periodo y debido a su principal aportador, que es el petróleo. Si bien la recaudación es importante ya que tiene tendencia creciente desde el 2006 hasta la fecha, considero que depende en gran medida del movimiento del producto principal que es el petróleo y otros secundarios como productos entre flores, cacao, banano, camarón que también influyen en el movimiento.

La recaudación caería significativamente si estos productos no tuvieran movimiento a nivel internacional y si la capacidad de gestión del SRI fuera más grande, la recaudación seria mucho mayor de lo que es actualmente.

Terminamos con las líneas generales en el periodo que comprende del 2013 en adelante, en el cual se observa una tendencia decreciente y muy significativa, nuevamente nos traslada al cuadrante negativo de la gráfica.

El ciclo económico que se viene luego del año 2013, es bastante difícil de llevar, si a esto le sumamos el tema político, tema que está muy quebrado en todas sus aristas, es un costo adicional y significativo para la economía del país, no solamente desde el punto de vista cuantitativo, es también desde el punto de vista cualitativo, disminuye el interés por invertir, por trabajar, por una producción competitiva, las personas tienen una incertidumbre del futuro, salvo nuevas pronunciaciones del gobierno actual.

Los ecuatorianos y ciertos gobernantes de los países de la región, somos fieles cumplidores de las políticas y recetas internacionales, más allá de que sea saludable o conveniente para nosotros, es una actuación muy común en el medio. El déficit fiscal del Ecuador es un tema 
muy serio y difícil de manejar, los recursos no son lo suficiente para cubrir el presupuesto y las facilidades para el endeudamiento no se hacen esperar.

Los organismos ofertantes de crédito, están a la expectativa de poner sus recursos en estados que de alguna manera garantizan el pago con sus principales recursos, como es el nuestro que tiene comprometido ciertas cantidades representativas de petróleo, lo cual se entiende que ciertos recursos futuros ya están gastados y hay que resolver los egresos de corto plazo.

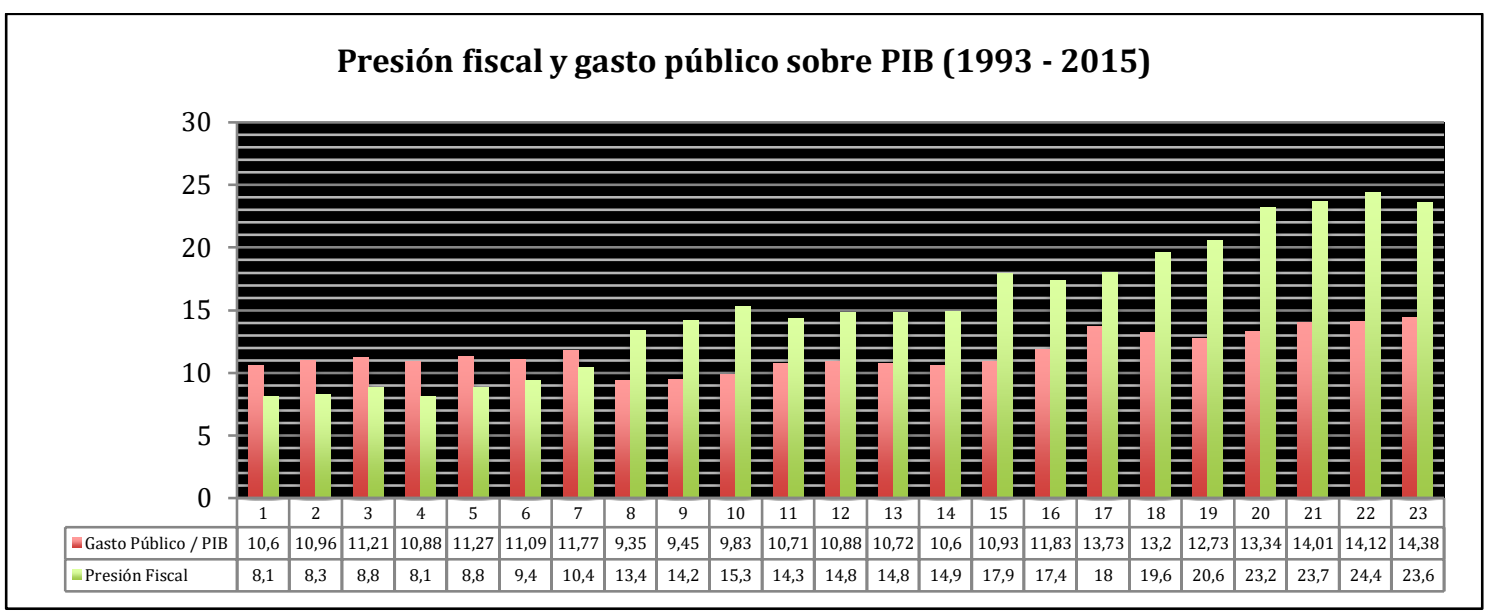

Figura 2. Gasto público sobre PIB (1990 - 2016)

Fuente: INEC (www.inec.gob.ec, 2008) - BID (https://www.iadb.org, 2008)

El gráfico dos muestra la presión fiscal y el gasto público sobre el PIB desde 1993 hasta el 2015, en el cual se puede observar una tendencia creciente de la presión fiscal, tendencia que proviene principalmente de dos causas, el incremento en la recaudación de impuestos y el decrecimiento del PIB, por lo tanto, el resultado se hace más representativo.

La teoría de Keynes está basada en que la demanda global mueve la actividad económica, misma que está formada por el consumo de los hogares, la demanda de bienes de inversión de las empresas, la demanda del sector público y por la demanda de los mercados internacionales, cuya ecuación se la expresa de la siguiente manera.

$$
D_{g}=C+I+G+E_{X}
$$

En la economía de un determinado país, básicamente tiene dos problemas fundamentales que son el desempleo y la inflación, mismos que se los quiere atacar con un juego de variables, en las cuales está inmersa la política fiscal. 
Para bajar el desempleo, se debería estimular la demanda global (Dg) mediante la estimulación de consumo de los hogares, esto implica bajar los impuestos para que las familias tengan más dinero destinado al consumo, como segunda gestión para estimular la demanda, es aumentar el gasto público en una medida que no incremente el déficit fiscal. Estas herramientas no deber servir para solucionar un problema y generar otro, dependiendo el ciclo económico que se encuentre, el gasto público será oportuno siempre y cuando tenga los recursos para aquello.

Observamos las gráficas 1,2 y 3 con el fin de relacionar las variables en apego al comportamiento teórico, y notamos que no tiene estricto cumplimiento. En niveles altos de desempleo, la carga impositiva o los impuestos directos no bajaron, el gasto público tiene una leve tendencia de crecimiento y los ciclos de la economía son bastante diferentes.

La posición de Keynes en relación al papel que debería jugar la política fiscal debe matizarse en el sentido de que su supuesta defensa a ultranza de la intervención pública sólo se justificaba en situaciones de desequilibrio, como la que caracterizó a un buen número de países occidentales tras la democracia (Crisis del 29) (Roura, 2010)

Si queríamos atacar el otro problema denominado inflación, las acciones eran diferentes a las anteriores, debemos bajar la demanda global, subiendo los impuestos para que baje el consumo y disminuyendo el gasto público; si bien, se observa que la inflación bajó, no creo que fue precisamente al cumplimiento de la teoría, ya que el gasto público subió levemente, los impuestos no subieron a pesar de que su recaudación si lo hizo, lo cual puede ser efecto de una mejor administración en la recaudación más que de incremento de la tasa impositiva.

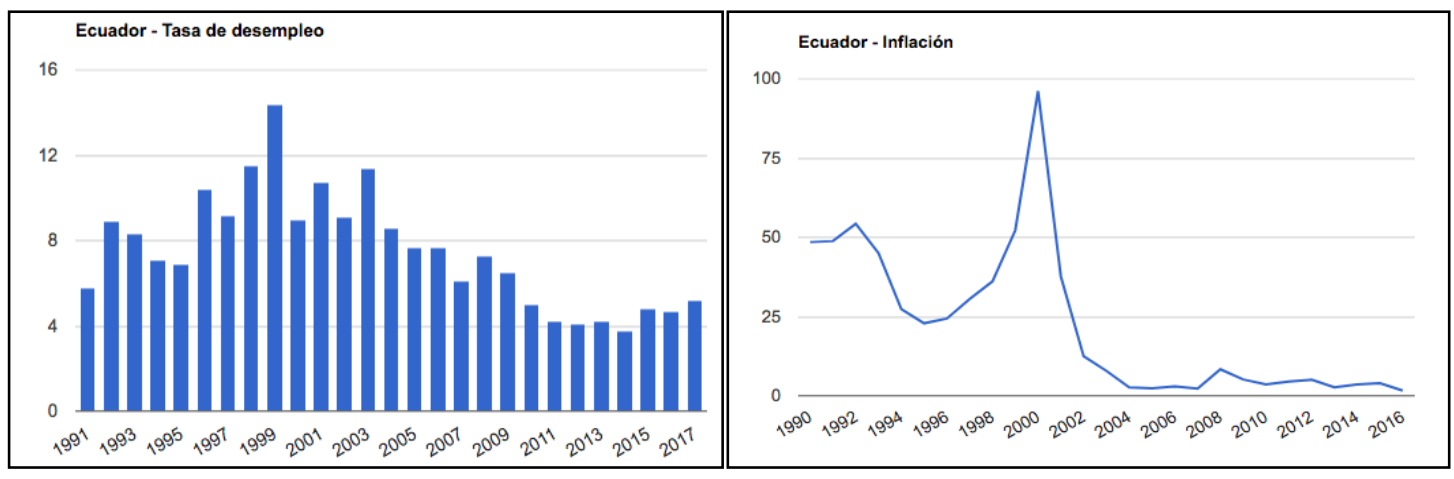

Figura 3. Tasa de desempleo e inflación Fuente: The global economy (2008) 
La presión fiscal del Ecuador del año 2015 comparada con las de los países de la región, está relativamente bordeando la media, año en el cual justamente se encuentran las curvas de variación del PIB del Ecuador y A.L y el Caribe, lo cual es bueno si comparamos con el rango menor y malo si comparamos con el rango mayor, esto significa que teniendo una misma tasa de variación del PIB, que por cierto es decreciente, tenemos algunos países que nos superaron en la efectividad recaudatoria de impuestos, como también tenemos algunos que les superamos en el año 2015.

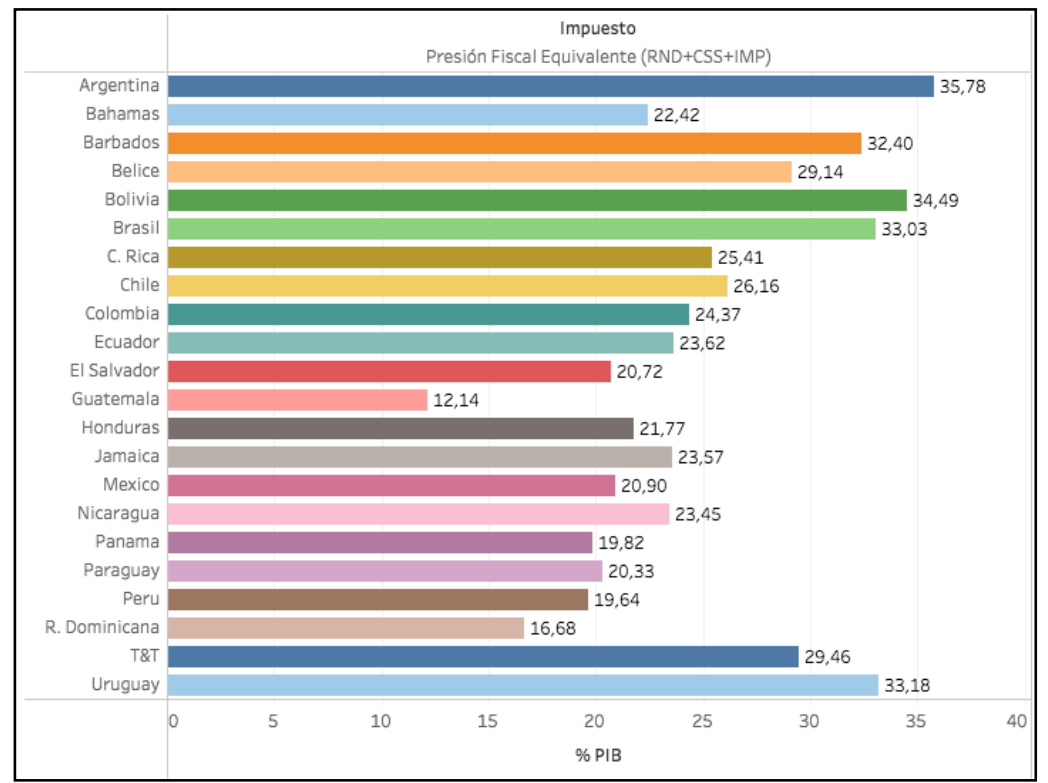

Figura 4. Presión fiscal (2015)

Fuente: BID (https://www.iadb.org, 2008)

\section{Conclusiones}

Ciertas estrategias que un momento y para un país determinado dieron buenos resultados, no significa que se las debe aplicar de manera generalizada para todos los países y más aún en momentos económicos diferentes, por lo que, si una teoría es buena para un país en un momento determinado, no significa que también lo será para otro país.

Las estrategias de expansión o restricción, debe ser ejecutadas sin afectar el mediano y largo plazo, no debemos trasladar un problema al futuro, para nuestro caso y según los datos expuestos en las gráficas, las curvas de inflación y desempleo son manejadas, pero a un costo de mayor endeudamiento, mayor déficit fiscal, de falta de recursos a corto plazo. Esto 
significa que, si no hay medidas correctivas adecuadas, el ciclo negativo está comenzando ya en estos años.

Si el país no cuenta con ventajas absolutas y representativas a nivel internacional, el crecimiento real y sostenido no es característica del Ecuador, seguimos dependiendo de los recursos naturales y en muchos de ellos sin un valor agregado representativo.

\section{Bibliografía}

Constitución de la República de Ecuador. (2008). Quito: Registro Oficial.

Wikipedia. (09 de 02 de 2008). https://es.wikipedia.org/wiki/Pol\%C3\%ADtica_fiscal.

Recuperado el 09 de 02 de 2008, de Wikipedia:

https://es.wikipedia.org/wiki/Pol\%C3\%ADtica_fiscal

Arthur Andersen. (1999). Diccionario de Economía y Negocios. Madrid, España: Espasa Calpe, S. A.

Roura, J. R. (2010). Política Económica. (C. Sánchez, Ed.) Madrid, España: INTERAMERICANA DE ESPAÑA.

https://www.cepal.org/es. (s.f.). https://www.cepal.org/es. Recuperado el 05 de 01 de 2008, de http://interwp.cepal.org/cepalstat/engine/index.html:

http://interwp.cepal.org/cepalstat/engine/index.html www.inec.gob.ec. (10 de 02 de 2008). Obtenido de http://www.ecuadorencifras.gob.ec/base-de-datos-censo-de-poblacion-y-vivienda/\#: http://www.ecuadorencifras.gob.ec/base-de-datos-censo-de-poblacion-y-vivienda/\#

https://www.iadb.org. (15 de 02 de 2008). https://www.iadb.org. Obtenido de https://publications.iadb.org/handle/11319/8601:

https://publications.iadb.org/handle/11319/8601

theglobaleconomy.com. (03 de 01 de 2008). theglobaleconomy.com. Obtenido de http://es.theglobaleconomy.com/Ecuador/Government_size/: http://es.theglobaleconomy.com/Ecuador/Government_size/

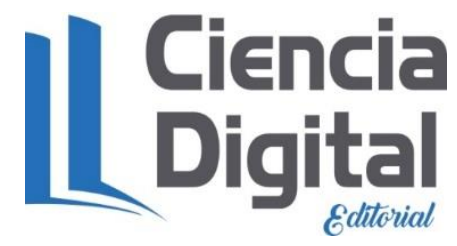




\section{PARA CITAR EL ARTÍCULO INDEXADO}

Chamba Bastidas, L., Colcha Ortiz, R., Moreno Albuja, M., \& Gavilanes Sagñay, M. (2019). Política tributaria y fiscal del Ecuador. Visionario Digital,3(3), 327-336. https://doi.org/10.33262/visionariodigital.v3i3.886

\section{LCigital}

El artículo que se publica es de exclusiva responsabilidad de los autores y no necesariamente reflejan el pensamiento de la Revista Visionario Digital.

El artículo queda en propiedad de la revista y, por tanto, su publicación parcial y/o total en otro medio tiene que ser autorizado por el director de la Revista Visionario Digital.
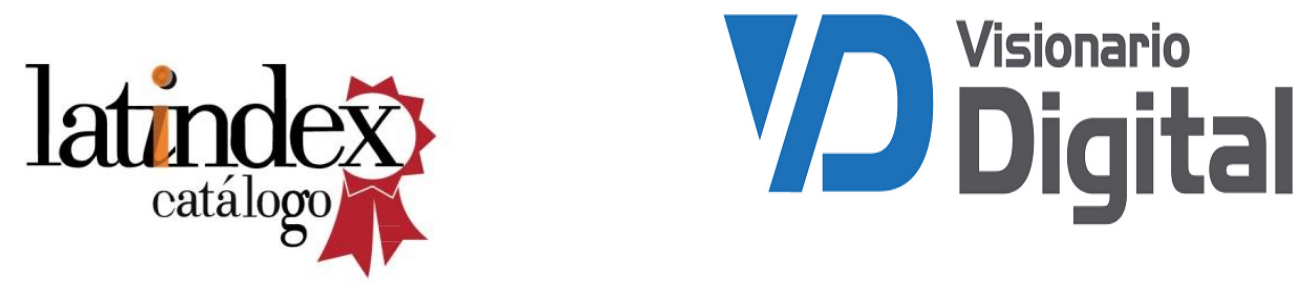